\title{
The relationship between iodine nutrition and thyroid disease in lactating women with different iodine intakes
}

\author{
Lixiang Liu ${ }^{1}$, Dandan Wang ${ }^{1}$, Peng Liu ${ }^{1}$, Fangang Meng ${ }^{1}$, Da Wen ${ }^{1}$, Qingzhen Jia ${ }^{2}$, Jun Liu ${ }^{3}$, \\ Xiaoye Zhang ${ }^{1}$, Peng Jiang ${ }^{1}$ and Hongmei Shen ${ }^{1 *}$ \\ ${ }^{1}$ Center for Endemic Disease Control, Chinese Center for Disease Control and Prevention, Harbin Medical University, Harbin \\ City, Heilongjiang Province 150081, People's Republic of China \\ ${ }^{2}$ Institute for Endemic Disease Prevention and Treatment of Shanxi Province, Linfen City, Shanxi Province O41000, People's \\ Republic of China \\ ${ }^{3}$ Center for Disease Control and Prevention, GuangXi Province, Nanning City, Guangxi Province 530028, People's Republic of China \\ (Submitted 1 April 2015 - Final revision received 9 July 2015 - Accepted 21 July 2015 - First published online 14 September 2015)
}

\begin{abstract}
Areas with low, adequate and excessive I content in water co-exist in China. Limited data are currently available on I nutrition and thyroid disease in lactating women and their breast-fed infants with different I intakes. This study aimed to evaluate I nutrition in both lactating women and their infants and the prevalence of thyroid disease in areas with different levels of I in water. From January to June 2014, a total of 343 healthy lactating women (excluding those taking anti-thyroid drugs or I supplements within a year of the study, consuming seafood at the time of the study or those diagnosed with congenital thyroid disease) from Beihai in Guangxi province and Jiajiazhuang, Yangcheng, Jicun and Pingyao townships in Shanxi province were selected. Compared with the I-sufficient group, median urinary I concentrations in both lactating women and infants as well as breast milk I levels were significantly lower in the I-deficient group $(P<0 \cdot 001)$. The prevalence of thyroid disease in lactating women, particularly subclinical hypothyroidism, was higher in the I-excess group than in the I-sufficient group $(P<0.05)$. In areas with excessive water I content, high thyroid peroxidase antibody and high thyroglobulin levels were risk factors for abnormal thyroid-stimulating hormone levels. Our data collectively suggest that excessive I intake potentially causes subclinical hypothyroidism in lactating women. Moreover, enhanced monitoring of I status is important to avoid adverse effects of I deficiency or excess, particularly in susceptible populations such as pregnant or lactating women and infants.
\end{abstract}

Key words: Iodine nutrition: Iodine excess: Iodine deficiency: Lactating women: Thyroid disease

I is a trace element that is essential for the synthesis of thyroid hormones that are required for normal growth, and therefore important for the development of newborns. Adequate dietary I intake is critical for susceptible populations with high I requirements, such as pregnant and lactating women as well as infants. Several studies have shown that I deficiency during crucial periods of development in infants leads to growth retardation, impaired hearing capacity and reduced cognitive function ${ }^{(1-3)}$. I intake is required not only for meeting the mother's own synthesis of thyroid hormones but also for transferring the hormones to the infant through breast milk (BM) for maintaining normal thyroid function as well as growth and development, particularly of the brain. This additional loss of $\mathrm{I}$ is the main reason underlying I deficiency in lactating women ${ }^{(4)}$. I deficiency can affect thyroid function during lactation, leading to adverse side-effects in the mother, and consequently pose a critical threat to growth and brain development in the breast-fed infant ${ }^{(1,2)}$. The current daily
I intake of $250 \mu \mathrm{g} / \mathrm{d}$ for lactating women has been recommended by $\mathrm{WHO} / \mathrm{ICCIDD} / \mathrm{UNICEF}$ to ensure that I deficiency does not occur in the postpartum period, and the I content of milk is sufficient for infants' requirements. In both lactating women and infants $<2$ years of age, median urinary iodine (MUI) concentration below $100 \mu \mathrm{g} / \mathrm{l}$ defines a population with I deficiency. However, limited data are available on the safe urinary iodine (UI) upper limit in lactating women ${ }^{(2)}$. Recent studies have shown that I excess in women (including lactating women) living in areas with high water I concentrations increases the risk of thyroid disease ${ }^{(5)}$. Other investigations have linked excessive I intake from BM to subclinical hypothyroidism in preterm Korean infants ${ }^{(6)}$. Accordingly, adequate I concentration in BM is considered particularly important for breast-fed infants to ensure optimal thyroid hormone storage and prevent neurological development impairment. Few studies to date have focused on the relationship between I intake and thyroid function in lactating women. The main objectives of

Abbreviations: BM, breast milk; FT4, free thyroxin; MUI, median urinary iodine; Tg, thyroglobulin; TgAb, thyroglobulin antibody; TPOAb, thyroid peroxidase antibody; TSH, thyroid-stimulating hormone; UI, urinary iodine.

* Corresponding author: H. Shen, fax +86 451866 57674, email shenhm119@126.com 
the present study were to clarify the status of I nutrition and the prevalence of thyroid disease among lactating women from three regions with different water I contents in China, to ascertain the relationship between I intake and the status of I nutrition as well as thyroid function in lactating women and their infants and to simultaneously provide reference data for appropriate disease control and prevention measures.

\section{Methods \\ Survey areas}

Villages for survey were selected based on the national surveillance data on areas with high water I contents, along with historical iodine deficiency disorders (IDD) surveillance data obtained in recent years ${ }^{(7,8)}$. The coastal area, Tieshangang district (Xinggang, Nankang and Yingpan Towns) of Beihai city, Guangxi province, was selected as the I-deficient region with median water iodine (MWI) $\leq 10 \mu \mathrm{g} / \mathrm{l}$ and with low coverage rates of iodised salt ${ }^{(9)}$. Luocheng village in Jiajiazhuang township and Xiaoguo village in Yangcheng township, both located in Fenyang City from Shanxi province, were selected as areas where residents have sufficient I nutrition $(50 \mu \mathrm{g} /$ $1 \leq \mathrm{MWI} \leq 150 \mu \mathrm{g} / \mathrm{l}$ ), representing the control group. Chengzi, Donghe, Guxianzhuang and Dongshe villages in Jicun township and Jinghua and Ninggu villages in Pingyao township (Fenyang city of Shanxi province) were selected as areas with high I water content $(\mathrm{MWI} \geq 300 \mu \mathrm{g} / \mathrm{l})^{(10)}$.

\section{Survey subjects}

From January to June 2014, a total of 343 lactating women (including 106, 104 and 133 subjects from the I-deficient, sufficient and excess regions, respectively) were recruited, excluding those taking anti-thyroid drugs or I supplements within a year of the study, consuming seafood at the time of the study and those with a family history of thyroid disease or having congenital thyroid disease. All the participants had lived in the region for $>5$ years. Infants whose breast-feeding occurred within a year of the study and with BM as the main source of food were selected.

\section{Survey indicators}

According to WHO recommendations, the following indicators were adopted to assess I status ${ }^{(2)}$ : MUI, goitre rate (GR), thyroid-stimulating hormone (TSH) and thyroglobulin (Tg). Given the limitations of these indicators, free tri-iodothyronine $\left(\mathrm{FT}_{3}\right)$, free thyroxin $\left(\mathrm{FT}_{4}\right)$, thyroglobulin antibody (TgAb) and thyroid peroxidase antibody (TPOAb) were included as novel indicators for testing. Reference values are presented in Table 1.

Thyroid volume, UI, water I, salt I, BM I and thyroid function of lactating women were additionally examined. These indicators were matched for individual subjects. As some participants did not provide all three samples (urine, BM and blood), the number of each sample type differed from the total number of subjects. Urine samples were additionally collected from infants of the lactating study participants.

This project was approved by the Ethical Review Board of Harbin Medical University. Written informed consent was obtained from all the participants before the survey was conducted.

\section{Survey methods, sample collections and measurements}

A standard questionnaire was designed to acquire demographic information including name, age, personal or family history of thyroid disease (including type of thyroid disease), intake of supplements containing I, smoking habits, economic income, source of drinking water and drinking duration. The questionnaire was administered face-to-face and conducted by well-trained staff.

A single-spot urine sample was collected in the morning in clean plastic tubes and stored at $4^{\circ} \mathrm{C}$ until batch-analysed for I content using the China Health Standard Method for Determination of Iodine in Urine by $\mathrm{As}^{3+}-\mathrm{Ce}^{4+}$ Catalytic Spectrophotometry ${ }^{(11)}$. Urine and standard samples $(250 \mu \mathrm{l})$ were digested with $1000 \mu \mathrm{l}\left(\mathrm{NH}_{4}\right)_{2} \mathrm{~S}_{2} \mathrm{O}_{8}(1.0 \mathrm{~mol} / \mathrm{l})$ at $100^{\circ} \mathrm{C}$ for $60 \mathrm{~min}$. Internal quality control samples for UI were provided by the Chinese National Reference Laboratory for Iodine Deficiency Disorders (NRLIDD).

In the pre-selected I-deficient area (Tieshangang district), each participant provided a water sample of at least $15 \mathrm{ml}$, whereas in the pre-selected I excess and sufficient villages, twenty-two water samples in total were collected from the central water supply. Water samples were stored at $4^{\circ} \mathrm{C}$ until

Table 1. Diagnostic criteria for thyroid disease

\begin{tabular}{|c|c|}
\hline Thyroid disease & Diagnostic criteria* \\
\hline Hypothyroxinaemia & $\mathrm{FT}_{4}<11.5 \mathrm{pmol} / \mathrm{l}$ and $\mathrm{TSH}$ within the normal range \\
\hline Overt hypothyroidism & $\mathrm{TSH}>4.20 \mathrm{mlU} / \mathrm{l}, \mathrm{FT}_{4}<11.5 \mathrm{pmol} / \mathrm{l}$ \\
\hline Subclinical hypothyroidism & $\mathrm{TSH}>4.20 \mathrm{mlU} / \mathrm{l}, \mathrm{FT}_{4}$ within the normal range \\
\hline Overt hyperthyroidism & $\mathrm{TSH}<0.27 \mathrm{mlU} / \mathrm{l}, \mathrm{FT}_{4}>22.7$ or $\mathrm{FT}_{3}>6.8 \mathrm{pmol} / \mathrm{l}$ \\
\hline Subclinical hyperthyroidism & $\mathrm{TSH}<0.27 \mathrm{mlU} / \mathrm{l}, \mathrm{FT}_{3}$ and $\mathrm{FT}_{4}$ within the normal range \\
\hline Autoimmune thyroiditis & TPOAb $>60 \mathrm{U} / \mathrm{ml}$ or TgAb $>60 \mathrm{U} / \mathrm{ml}$ with overt or subclinical hypothyroidism \\
\hline High serum autoantibody values & TPOAb $>60 \mathrm{U} / \mathrm{ml}$ or TgAb $>60 \mathrm{U} / \mathrm{ml}$ \\
\hline Goitre & Thyroid volume $18 \mathrm{ml}$ (female)† \\
\hline
\end{tabular}

$\mathrm{FT}_{3}$, free tri-iodothyronine; $\mathrm{FT}_{4}$, free thyroxin; $\mathrm{Tg}$, thyroglobulin; TgAb, thyroglobulin antibody; TPOAb, thyroid peroxidase antibody; TSH, thyroidstimulating hormone.

* Reference values: $\mathrm{FT}_{4}, 11.5-22.7 \mathrm{pmol} / \mathrm{l} ; \mathrm{TSH}, 0.27-4.2 \mathrm{mlU} / \mathrm{l} ; \mathrm{FT}_{3}$, 3.1-6.8 pmol//; TPOAb, 0-60 U/ml; TgAb, 0-60 U/ml.

$\dagger$ Based on the diagnostic criterion of endemic goitre ${ }^{(13)}$. 
analysis. The I concentration of drinking water was determined using the method recommended by NRLIDD, the Chinese centre for disease control and prevention.

The I content in table salt from households of lactating participants was determined using the general test method of the salt industry ${ }^{(12)}$. Each participant provided a table salt sample of at least $50 \mathrm{~g}$. The standard salt I content in Guangxi province and Shanxi province is $25 \mathrm{mg} / \mathrm{kg}$. Salt type was classified into the following categories: non-iodised salt (salt I content $<5 \mathrm{mg} / \mathrm{kg}$ ), qualified iodised salt $(18 \mathrm{mg} / \mathrm{kg} \leq$ salt I content $\leq 33 \mathrm{mg} / \mathrm{kg})$ and unqualified iodised salt $(5 \mathrm{mg} / \mathrm{kg}<$ salt I content $<18 \mathrm{mg} / \mathrm{kg}$ or $>33 \mathrm{mg} / \mathrm{kg}$ ).

An aliquot of at least $5 \mathrm{ml} \mathrm{BM}$ was collected from each lactating subject for determining the I content and stored at $-20^{\circ} \mathrm{C}$ until analysis. I content was determined using arsenic-cerium catalytic spectrophotometry ${ }^{(13)}$, the National Standard method of the Chinese Ministry of Health. BM and standard samples $(500 \mu \mathrm{l})$ were ashed with $1000 \mu \mathrm{l}-\mathrm{K}_{2} \mathrm{CO}_{3}-\mathrm{NaCl}$ and $1000 \mu \mathrm{l}-\mathrm{ZnSO}_{4}-\mathrm{KClO}_{3}$ in a Muffle furnace at $600^{\circ} \mathrm{C}$ for $4 \mathrm{~h}$ after drying and carbonisation.

Thyroid ultrasonography was performed by an experienced examiner using a $7.5 \mathrm{MHz}$ transducer (mainly measuring the thyroid volume, nodule diameter and echo). Thyroid lobe volume was calculated by measuring the depth $(d)$, width $(w)$ and length $(l)$ of each lobe using the following formula: $V(\mathrm{ml})=$ $0.479 \times d \times w \times l(\mathrm{~mm}) / 1000$, and was recorded as the sum of both lobes. The normal volume for female adults was $<18 \mathrm{ml}^{(14)}$.

Blood samples $(5-6 \mathrm{ml})$ were collected from the cubital vein of lactating women. Serum samples were prepared by centrifugation at $3000 \mathrm{rpm}$ for $10 \mathrm{~min}$ after allowing the samples to stand for $30 \mathrm{~min}$ and were subsequently frozen at $-80^{\circ} \mathrm{C}$ until analysis. TSH, $\mathrm{FT}_{3}, \mathrm{FT}_{4}$, TPOAb and TgAb levels were determined using the chemiluminescent immunoassay (Bayer Healthcare Company, Siemens Medical Solutions Diagnostics). Tg levels were measured using electrochemiluminescence immunoassay, Elecsys and Cobas diagnostics (Bayer Healthcare Company, Roche).

\section{Statistical analysis}

All the data were recorded with EpiData3.1. Data processing and statistical analyses were performed using SPSS software (version 13.0). Normally distributed data were expressed as mean values and standard deviations, whereas non-normally distributed data were expressed as median with the 25th and 75 th percentiles. ANOVA was used to compare normally distributed data, and the Mann-Whitney test was used to compare non-normally distributed data among the groups. The proportions among the three groups were analysed with the $\chi^{2}$ test. Spearman's method was used for correlation analysis of the variables. Binary logistic regression analysis was applied to calculate the OR and 95\% CI for abnormal TSH of lactating women from the three areas. Data were considered statistically significant at $P<0 \cdot 05$.

\section{Results}

Subjects

A total of 343 lactating women were recruited for the survey. Demographic characteristics of the study subjects are presented in Table 2. Water I contents varied significantly among the three groups. No significant differences were observed with regard to age, height, weight and smoking habits among subjects from all three groups.

\section{lodine nutritional status}

MUI values of lactating women were 51.30, 282.42 and $822.51 \mu \mathrm{g} /$ 1 for the I-deficient, sufficient and excess groups, respectively, as shown in Table 3. Compared with the I-sufficient group, MUI values were significantly lower in the I-deficient group $(Z=-11.566 ; P=0.000)$ and significantly higher in the I-excess group $(Z=-10 \cdot 002 ; P=0 \cdot 000)$. In parallel, MUI of breast-fed infants and median I content in BM differed significantly among the three groups.

Positive correlations among BM I and UI of lactating women as well as UI of their breast-fed infants are depicted on scatter plots in Fig. 1.

\section{Assessment of thyroid hormones and antibodies}

Compared with the I-deficient group, the $\mathrm{FT}_{3}$ levels of lactating women were significantly higher in the I-sufficient and excess groups (5.25 and $5.25 v .4 .79 \mathrm{pmol} / \mathrm{l} ; P=0.049,0.020)$.

We additionally observed a trend for increased serum $\mathrm{FT}_{4}$ concentrations with increasing water I content. In the I-excess group, $\mathrm{FT}_{4}$ levels of lactating women were significantly higher compared with the I-deficient group (14.10 v. $12.71 \mathrm{pmol} / \mathrm{l}$; $P=0 \cdot 010)$

Median TSH concentrations of lactating women in the I-deficient group were significantly lower than those in the I-sufficient group $(Z=-6.828 ; P=0.000)$ and were higher in the I-excess group compared with the I-sufficient group ( $Z=-1 \cdot 864 ; P=0 \cdot 062$ ), although this difference was not statistically significant.

Serum Tg levels varied markedly among the three groups and were higher in the I-deficient and excess groups compared with the I-sufficient group (18.36 and $16.25 v .10 .53 \mu \mathrm{g} / \mathrm{l} ; Z=-4.286$, $P=0.000 ; Z=-2.579, P=0.01)$.

We observed no significant differences in positive rates for TgAb, TPOAb and the combined antibodies among the three groups $(7 \cdot 55,13.46$ and $15.79 \%$ for TgAb; 13.21, 9.62 and $12.79 \%$ for TPOAb; and 5.66, 6.73 and $9.02 \%$ for TgAb and TPOAb for the I-deficient, sufficient and excess groups, respectively; Table 4).

Non-linear correlations among TSH and Tg and MUI of lactating women were observed, their relationships are depicted on scatter plots in Fig. 2.

\section{Thyroid diseases}

Compared with the I-sufficient group, the prevalence of subclinical hypothyroidism was significantly higher $\left(\chi^{2}=4.486\right.$; $P=0.034)$ in the I-excess group. However, we observed no significant differences in the prevalence of other thyroid diseases examined among the three groups (Table 5). 
Table 2. Demographic characteristics of lactating women from three different water iodine groups (Normally distributed mean values and standard deviations; non-normal distributed 25th and 75th percentiles)

\begin{tabular}{|c|c|c|c|c|c|c|}
\hline \multirow[b]{2}{*}{ Characteristics } & \multicolumn{2}{|c|}{ Beihai (I-deficient areas) } & \multicolumn{2}{|c|}{$\begin{array}{l}\text { Yangcheng and Jiajiazhuan } \\
\text { (I-sufficient areas) }^{\star}\end{array}$} & \multicolumn{2}{|c|}{$\begin{array}{l}\text { Pingyao and Jicun } \\
\text { (I-excess areas) }\end{array}$} \\
\hline & Mean & SD & Mean & SD & Mean & SD \\
\hline$n \dagger$ & \multicolumn{2}{|c|}{106} & \multicolumn{2}{|c|}{104} & \multicolumn{2}{|c|}{133} \\
\hline Age (years) & 26.59 & 4.88 & 25.53 & 3.60 & $25 \cdot 49$ & $3 \cdot 30$ \\
\hline Height $(m) \ddagger$ & 1.57 & 0.06 & 1.59 & 0.06 & 1.59 & 0.05 \\
\hline Weight (kg)‡ & $58 \cdot 53$ & 6.97 & $60 \cdot 26$ & 11.89 & $62 \cdot 07$ & 13.02 \\
\hline Smoking & \multicolumn{2}{|c|}{ None } & \multicolumn{2}{|c|}{ None } & \multicolumn{2}{|c|}{ None } \\
\hline MWI $(\mu \mathrm{g} / \mathrm{l})$ & \multicolumn{2}{|c|}{$2 \cdot 2 \S$} & \multicolumn{2}{|c|}{57.5} & \multicolumn{2}{|c|}{464.8} \\
\hline P25-P75 & \multicolumn{2}{|c|}{$1.18-4.05$} & \multicolumn{2}{|c|}{$19.00-83.33$} & \multicolumn{2}{|c|}{$339.60-508.73$} \\
\hline I content of iodised salt (mg/kg) & 20.94 & 2.92 & 11.90 & 4.71 & 11.73 & 3.77 \\
\hline Coverage of household use of qualified iodised salt (\%) & \multicolumn{2}{|c|}{14.29} & \multicolumn{2}{|c|}{8.33} & \multicolumn{2}{|c|}{2.02} \\
\hline
\end{tabular}

MWI, median water iodine.

* The control group.

† The sample sizes.

$\ddagger$ Water I content varied significantly among the three groups $(P<0.01)$.

Table 3. Status of iodine nutrition in lactating women and infants from the three water iodine groups* (Medians, sample sizes and 25th to 75th percentiles)

\begin{tabular}{|c|c|c|c|c|c|c|c|c|c|}
\hline & \multicolumn{3}{|c|}{$\begin{array}{c}\text { Beihai } \\
\text { (I-deficient areas) }\end{array}$} & \multicolumn{3}{|c|}{$\begin{array}{l}\text { Yangcheng and Jiajiazhuang } \\
\text { (I-sufficient areas) } \dagger\end{array}$} & \multicolumn{3}{|c|}{$\begin{array}{l}\text { Pingyao and Jicun } \\
\text { (I-excess areas) }\end{array}$} \\
\hline & Median & $n$ & P25-P75 & Median & $n$ & P25-P75 & Median & $n$ & P25-P75 \\
\hline MUI of lactating women $(\mu \mathrm{g} / \mathrm{l})$ & $51 \cdot 30$ & $113 \ddagger$ & $28 \cdot 05-73 \cdot 65$ & 282.42 & 98 & $176 \cdot 77-386 \cdot 98$ & $822 \cdot 51$ & $125 \S$ & $558 \cdot 45-1508 \cdot 00$ \\
\hline MUI of infants $(\mu \mathrm{g} / \mathrm{l})$ & $64 \cdot 85$ & $28 \|$ & $33 \cdot 53-105 \cdot 7$ & $427 \cdot 01$ & 90 & $269 \cdot 55-983 \cdot 37$ & $1222 \cdot 41$ & $124 \|$ & $786 \cdot 93-1995 \cdot 00$ \\
\hline Median I content in breast milk $(\mu \mathrm{g} / \mathrm{l})$ & 41.47 & $103 \|$ & $26 \cdot 39-64 \cdot 38$ & $346 \cdot 11$ & 91 & $208 \cdot 66-449 \cdot 46$ & $942 \cdot 33$ & $99 \|$ & $739 \cdot 26-1359 \cdot 06$ \\
\hline
\end{tabular}

MUI, median urinary iodine.

* Test for difference among the three groups: Mann-Whitney test.

$\dagger$ The control group.

$\ddagger$ Compared with the control group, MUI values were significantly lower in the I-deficient group $(Z=-11.566 ; P=0.000)$.

$\S$ Compared with the control group, MUI values were significantly higher in the l-excess group $(Z=-10.002 ; P=0.000)$.

॥ MUI of breast-fed infants and median I content in breast milk differed significantly among the three groups $(P<0.01)$.

\section{Analysis of risk factors for abnormal thyroid-stimulating} hormone in lactating women

Rates of abnormal TSH values (including cases of hypothyroidism, subclinical hypothyroidism, hyperthyroidism and subclinical hyperthyroidism) differed significantly among the three groups $\left(\chi^{2}=14 \cdot 511 ; P=0 \cdot 001\right)$. Further analysis of associated factors revealed that water I, positive rates of TPOAb and TgAb, serum Tg level and UI are risk factors for abnormal TSH. Logistic regression analysis showed that high water I areas (OR 2.295; $95 \%$ CI 1.067, 4.932; $P=0.033$ ), high TPOAb (OR 11.033; $95 \%$ CI 5.033, 24.185; $P=0 \cdot 001)$ and high Tg levels (OR 1.014; $95 \%$ CI $1.005,1.024 ; P=0.002)$ are risk factors for abnormal TSH levels (Table 6).

\section{Discussion}

Thyroid hormones play an important role in growth and development of the human body, particularly in the brain. The I intake of breast-fed infants relies solely on concentrations in $\mathrm{BM}$, the only source of I after birth, to meet the infant's requirements for the synthesis of thyroid hormones ${ }^{(15)}$. Thus, brain development in infants is positively associated with the status of I nutrition and thyroid function of the mother. Previously, IDD were widespread in China. In recent years, IDD has been effectively controlled using the Universal Salt Iodization (USI) programme. However, pregnant, lactating women and women of reproductive age remain at higher risk ${ }^{(16-18)}$. In China, thirty-one million people are currently living in high water $\mathrm{I}$ areas ${ }^{(7)}$. Considering the complicated geographical environment of China, we evaluated the status of I nutrition and the prevalence of thyroid disease among lactating women in three regions with different water I contents.

This study aimed to assess I nutrition in lactating women in areas with different water I contents. Areas where salt was rarely used were selected to avoid interference from I in salt, thus ensuring minimal contribution to UI. Coverage of qualified iodised salt was $14.29,8.33$ and $2.02 \%$ in deficient, adequate and excess areas, respectively. Notably, coverage of qualified iodised salt in Beihai was rather low, as cheaper sea salt was used by the residents in this coastal city. The use of iodised salt has been forbidden in I-excess areas ${ }^{(19)}$. Both Jiajiazhuang and Yangcheng townships are in Fenyang city, located adjacent to high water I areas; therefore, the I content of iodised salt and 

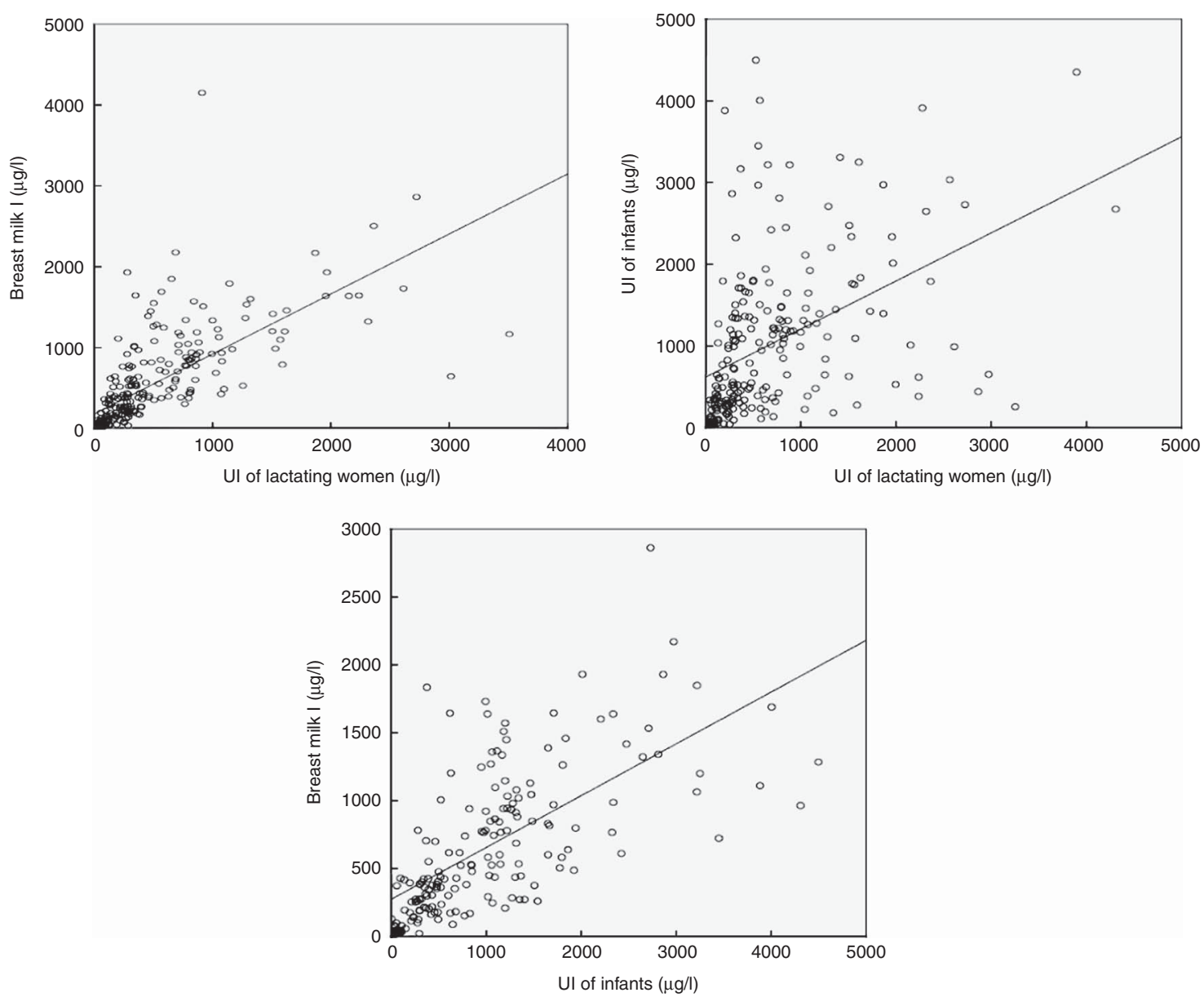

Fig. 1. Positive correlations among breast milk (BM) I, urinary iodine (UI) concentrations of lactating women and UI concentrations of their infants on a scatter plot. Positive correlations are evident between $\mathrm{BM} I$ and UI of lactating women, $\mathrm{UI}$ of infants and $\mathrm{UI}$ of lactating women and BM I and UI of infants $(r 0.879, P=0.000 ; r 0.623$, $P=0.000 ; r 0.733, P=0.000$

coverage of qualified iodised salt were relatively low owing to the usage of non-iodised salt.

A number of international researchers and the WHO have recommended that I nutrition from $\mathrm{BM}$ is optimal at concentrations of $100-200 \mu \mathrm{g} / 1$ to ensure normal development in infants $^{(20-22)}$. When the MUI of infants $<2$ years of age is above $100 \mu \mathrm{g} / \mathrm{l}$, the current I nutrition status of the population is considered adequate. In our study, the median I concentration in water from Beihai in Guangxi province was only $2 \cdot 2 \mu \mathrm{g} / \mathrm{l}$, clearly reflecting the lack of I in the external environment. In addition, median BM I was $41.47 \mu \mathrm{g} / \mathrm{l}$ and MUI was $64.85 \mu \mathrm{g} / \mathrm{l}$ in infants, which were lower than the recommended values. These results clearly reveal deficient I nutritional status among lactating women and infants from Beihai. In contrast, MUI was as high as 822.51 and $1222.41 \mu \mathrm{g} / \mathrm{l}$, respectively, in lactating women and infants from Pingyao and Jicun villages in Shanxi province, indicating excessive I nutritional status in these cases. The above findings support the recommendation that UI concentration should be monitored in lactating women and infants, especially for populations living in high or low water I areas.

Owing to loss of I in BM and urine during lactation, dietary I requirements are increased in lactating women, and
I metabolism is enhanced with increasing I intake. The BM I content increased with UI concentration in lactating women in our study. The I intake of breast-fed infants relies solely on concentrations in BM. BM I and UI of breast-fed infants were positively correlated, confirming that the I nutrition status of infants is affected by the I nutrition status of their mothers ${ }^{(23,24)}$.

Previous studies have reported increasing TSH levels with I intake in adults ${ }^{(18,25)}$. Consistent with earlier data, we observed an increase in the median concentration of TSH with increasing water I concentrations in lactating women from three regions with different water I contents. The $\mathrm{FT}_{3}, \mathrm{FT}_{4}$, TSH and UI concentration were low in Beihai, an area with low I levels in drinking water. However, GR was $>5 \%$, which was consistent with a report documenting high GR in lactating women in I-deficient areas ${ }^{(26)}$. Accordingly, we speculated that Beihai city is a mild I-deficient area with a low prevalence of thyroid disease.

We compared the utility of TSH and Tg as biomarkers of I status in lactating women using two methods. (1) Groups were classified based on the three different I nutrition areas (Table 4). In this case, TSH increased with the water I content. However, median Tg levels of lactating women in both I-deficient and 
Table 4. Thyroid parameters of lactating women in the three water iodine groups* (Numbers and percentages; mean values and standard deviations; medians and 25th to 75th percentiles)

\begin{tabular}{|c|c|c|c|c|c|c|}
\hline & \multicolumn{2}{|c|}{ Beihai (I-deficient areas) } & \multicolumn{2}{|c|}{ Yangcheng and Jiajiazhuang (I-sufficient areas)† } & \multicolumn{2}{|c|}{ Pingyao and Jicun (I-excess areas) } \\
\hline & $n$ & $\%$ & $n$ & $\%$ & $n$ & $\%$ \\
\hline$n \ddagger$ & \multicolumn{2}{|c|}{106} & \multicolumn{2}{|c|}{104} & \multicolumn{2}{|c|}{133} \\
\hline \multicolumn{7}{|l|}{$\mathrm{FT}_{3}(\mathrm{pmol} / \mathrm{l})$} \\
\hline Mean & \multicolumn{2}{|c|}{4.79} & \multicolumn{2}{|c|}{$5 \cdot 25$} & \multicolumn{2}{|c|}{$5 \cdot 25$} \\
\hline SD & \multicolumn{2}{|c|}{1.36} & \multicolumn{2}{|c|}{$1.04 \S$} & \multicolumn{2}{|c|}{$1.54 \|$} \\
\hline \multicolumn{7}{|l|}{$\mathrm{FT}_{4}(\mathrm{pmol} / \mathrm{l})$} \\
\hline Mean & \multicolumn{2}{|c|}{$12 \cdot 71$} & \multicolumn{2}{|c|}{$13 \cdot 47$} & \multicolumn{2}{|c|}{$14 \cdot 10$} \\
\hline SD & \multicolumn{2}{|c|}{3.11} & \multicolumn{2}{|c|}{2.52} & \multicolumn{2}{|c|}{$4.14 \pi$} \\
\hline \multicolumn{7}{|l|}{ TSH (mlU/l) } \\
\hline Median & \multicolumn{2}{|c|}{$1.08^{\star \star}$} & \multirow{2}{*}{\multicolumn{2}{|c|}{$\begin{array}{c}2 \cdot 17 \\
1.44-3 \cdot 11\end{array}$}} & \\
\hline P25-P75 & \multicolumn{2}{|c|}{$0.76-1.57$} & & & \multirow{2}{*}{\multicolumn{2}{|c|}{$1.60-3.51$}} \\
\hline $\operatorname{Tg}(\mu \mathrm{g} / \mathrm{l})$ & & & \multicolumn{2}{|c|}{ (1) } & & \\
\hline Median & \multirow{2}{*}{\multicolumn{2}{|c|}{$\begin{array}{c}18 \cdot 36+\dagger \\
12 \cdot 10-30 \cdot 73\end{array}$}} & \multirow{2}{*}{\multicolumn{2}{|c|}{$\begin{array}{c}10 \cdot 53 \\
6 \cdot 34-19.73\end{array}$}} & \multirow{2}{*}{\multicolumn{2}{|c|}{$\begin{array}{r}16 \cdot 25 \ddagger \ddagger \\
7.77-28.58\end{array}$}} \\
\hline P25-P75 & & & & & & \\
\hline TgAb-positive rate & 8 & 7.55 & 14 & 13.46 & 21 & $15 \cdot 79$ \\
\hline TPOAb-positive rate & 14 & $13 \cdot 21$ & 10 & $9 \cdot 62$ & 17 & $12 \cdot 79$ \\
\hline $\operatorname{TgAb}$ and TPOAb-positive rate & 6 & $5 \cdot 66$ & 7 & $6 \cdot 73$ & 12 & 9.02 \\
\hline
\end{tabular}

$\mathrm{FT}_{3}$, free tri-iodothyronine; $\mathrm{FT}_{4}$, free thyroxin; Tg, thyroglobulin; TgAb, thyroglobulin antibody; TPOAb, thyroid peroxidase antibody; TSH, thyroid-stimulating hormone.

* Differences among the three groups were analysed by ANOVA or the Mann-Whitney test.

$\dagger$ The control group.

‡ The sample sizes.

$\S$ Compared with the I-deficient group, the $\mathrm{FT}_{3}$ levels of lactating women were significantly higher in the I-sufficient groups $(P=0.049)$.

II Compared with the I-deficient group, the $\mathrm{FT}_{3}$ levels of lactating women were significantly higher in the l-excess groups $(P=0.020)$.

II In the I-excess group, $\mathrm{FT}_{4}$ levels of lactating women were significantly higher compared with the I-deficient group (14.10 v. 12.71 pmol/l; $\left.P=0.010\right)$

${ }_{\star *}$ Median TSH concentrations of lactating women in the I-deficient group were significantly lower compared with the $\mathrm{I}$-sufficient group $(Z=-6 \cdot 828, P=0.000)$.

†† Serum Tg levels were higher in the I-deficient group than the I-sufficient group $(Z=-4.286, P=0.000)$.

㧊 Serum Tg levels were higher in the I-excess group than the I-sufficient group $(Z=-2.579, P=0.01)$.

excess groups (18.36 and $16 \cdot 25 \mu \mathrm{g} / \mathrm{l}$, respectively) were significantly higher than that in the I-sufficient group $(10.53 \mu \mathrm{g} / \mathrm{l})$, showing lower values in the middle and higher values on both sides (approximate 'V shape'). (2) Groups were classified based on the different UI concentration levels (Fig. 2). In this study, the TSH level was increased and subsequently decreased with increased levels of MUI, similar to data from a previous study ${ }^{(27)}$ (approximate inverted ' $U$ curve'). In children, a 'U curve' relationship between TSH and UI has been reported ${ }^{(27)}$. However, no obvious correlation was evident between median $\mathrm{Tg}$ and MUI. I nutrition in children is sufficient at $\mathrm{Tg}<13 \mu \mathrm{g} / \mathrm{l}$. A 'U curve' relationship has been demonstrated between UI concentration and median Tg level in children, suggesting that $\mathrm{Tg}$ is a useful biomarker for I deficiency and excess I intake ${ }^{(28,29)}$. However, we assumed that children and lactating women are different populations, the latter being affected by the external environment, metabolism of the body itself, diet and small sample size, and further research on a larger scale is warranted. Our preliminary data indicate that TSH and Tg are not suitable as biomarkers for evaluating I nutrition in lactating women.

In our comparative analysis of I-deficient and sufficient areas, increased prevalence of thyroid disease (hypothyroidism, subclinical hypothyroidism, hyperthyroidism and subclinical hyperthyroidism, referred to as abnormal TSH) of lactating women was observed in I-excess areas. In high water I areas, high TPOAb and high Tg levels were determined as risk factors for abnormal TSH among lactating women. However, the influence of I deficiency on abnormal TSH was not established in this study, possibly because only mild I deficiency was present in our survey area of Beihai, Guangxi. Notably, our results are analogous with previous findings of increased incidence of subclinical hypothyroidism in pregnant women under high I conditions ${ }^{(30,31)}$. The prevalence of hypothyroxinaemia was evaluated as $14.42 \%$ in I-sufficient areas, compared with an earlier study showing $10.54 \%$ in six I-sufficient areas in China ${ }^{(27)}$. Taking into consideration the complicated geographical environment of China, household coverage of adequately iodised salt and demographic characteristics in the scope of the current survey, we propose that excessive I intake may lead to thyroid disease, especially subclinical hypothyroidism.

I deficiency results in a wide spectrum of adverse effects throughout the life cycle. Among these, the effect of I deficiency on infant intellectual development is of the greatest concern. No clear results on the association between loss of intelligence and I excess have been obtained to date. In this study, we focused on the status of I nutrition in relation to the prevalence of thyroid disease in lactating women. Urine samples were additionally collected from breast-fed infants to determine UI levels (as urine samples were difficult to collect for infants, we were unable to obtain all the counterpart samples from both lactating women and their infants). Moreover, the infants examined in this study were too young to obtain blood samples, and therefore we planned to use experimental animals for further research. Another limitation of this study was the lack of dietary assessment in individuals (e.g. water consumption was not measured).

Monitoring the I status of schoolchildren alone is not adequate. The Ministry of Health in China recommended careful monitoring of the I nutrition status in lactating women as part of the state monitoring for preventing and controlling tasks 

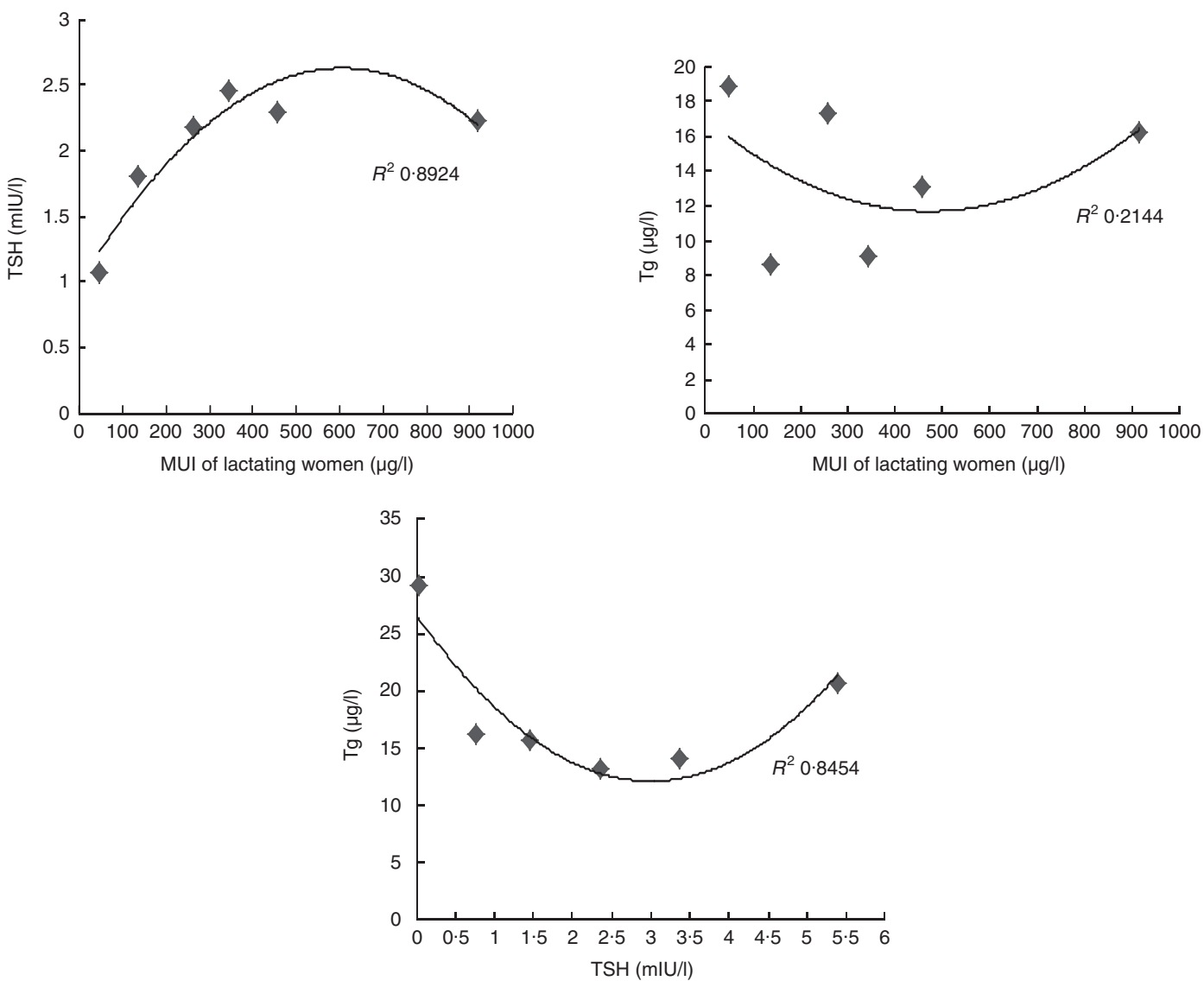

Fig. 2. Median urinary iodine (MUI) was divided into six groups (<100, 100-199.9, 200-299.9, 300-399.9, 400-499.9 and $>500 \mu \mathrm{mg} / \mathrm{l})$. Thyroid-stimulating hormone (TSH) levels tended to increase and subsequently decrease with increased levels of MUI. TSH was divided into six groups $(<0.27,0.27-0.99,1.00-1.99,2.00-2.99$, $3.00-4.19$ and $>4.20 \mathrm{mIU} / \mathrm{l})$. Thyroglobulin values showed a decrease and subsequent increase with increased levels of TSH, forming a ' $U$ curve' relationship in lactating women.

Table 5. Prevalence of thyroid disease among lactating women from the different water I groups ${ }^{\star}$ (Number of cases and percentages)

\begin{tabular}{|c|c|c|c|c|c|c|}
\hline & \multicolumn{2}{|c|}{ Beihai (I-deficient areas) } & \multicolumn{2}{|c|}{ Yangcheng and Jiajiazhuang (I-sufficient areas)† } & \multicolumn{2}{|c|}{$\underline{\text { Pingyao and Jicun (I-excess areas) }}$} \\
\hline & $n$ & $\%$ & $n$ & $\%$ & $n$ & $\%$ \\
\hline$n$ & \multicolumn{2}{|c|}{106} & \multicolumn{2}{|c|}{104} & \multicolumn{2}{|c|}{133} \\
\hline Hypothyroxinaemia (\%) & 18 & 16.98 & 15 & 14.42 & 14 & $10 \cdot 53$ \\
\hline Hypothyroidism & 4 & 3.77 & 4 & 3.85 & 9 & 6.77 \\
\hline Subclinical hypothyroidism & 1 & 0.94 & 6 & $5 \cdot 77$ & 19 & $14 \cdot 29 \ddagger$ \\
\hline Hyperthyroidism & 2 & 1.89 & 4 & 3.85 & 6 & 4.51 \\
\hline Subclinical hyperthyroidism & 2 & 1.89 & 2 & 1.92 & 2 & 1.50 \\
\hline Autoimmune thyroiditis & 4 & 3.77 & 5 & 4.81 & 9 & 6.77 \\
\hline High serum autoantibody values & 16 & $15 \cdot 09$ & 17 & $16 \cdot 83$ & 26 & $19 \cdot 55$ \\
\hline Goitre prevalence (\%) & 6 & $5 \cdot 66$ & 2 & 0.96 & 1 & 0.75 \\
\hline Thyroid nodules prevalence (\%) & 17 & $16 \cdot 04$ & 23 & $22 \cdot 12$ & 24 & $18 \cdot 05$ \\
\hline
\end{tabular}

* Differences among the three groups were analysed by $x^{2}$ test.

$\dagger$ The control group.

¥ Compared with the I-sufficient group, the prevalence of subclinical hypothyroidism was significantly higher $\left(X^{2}=4.486 ; P=0.034\right)$ in the I-excess group.

programme in $2011^{(8)}$. UI concentrations among lactating women were monitored in thirty-one provinces and corps. According to the monitoring outcomes, MUI values among most lactating women were at the national level. However, both water I content and household coverage of adequately iodised salt were low in Beihai, Guangxi, where the prevalence of thyroid disease was not high but UI levels were low in lactating women and infants. These results clearly indicate that the status of I nutrition in lactating women and infants remains deficient in a number of regions in China. To ensure that brain development in infants is not affected as a result of I deficiency, the relevant governmental departments and the salt industry should 
Table 6. Association of abnormal thyroid-stimulating hormone $(\mathrm{TSH})^{\star}$ of lactating women with water $I$ and other risk factors

(Odds ratios and $95 \%$ confidence intervals)

\begin{tabular}{lrll}
\hline Factor & OR $\dagger$ & \multicolumn{1}{c}{$95 \% \mathrm{Cl} \dagger$} & $P$ \\
\hline Water I $(\mu \mathrm{g} / \mathrm{l})$ & & & \\
$50 \mu \mathrm{g} / \mathrm{MWl} \leq 150 \mu \mathrm{g} / \mathrm{l}$ & 1.000 & & \\
$\mathrm{MWI} \leq 10 \mu \mathrm{g} / \mathrm{l}$ & 0.436 & $0.164,1.155$ & 0.095 \\
$\quad \mathrm{MWl} \geq 300 \mu \mathrm{g} / \mathrm{l}$ & 2.295 & $1.067,4.932$ & 0.033 \\
$\mathrm{TgAb}-$ positive rate & 0.915 & $0.326,2.566$ & 0.865 \\
TPOAb-positive rate & 11.033 & $5.033,24.185$ & 0.001 \\
Tg & 1.014 & $1.005,1.024$ & 0.002 \\
Median urinary I $(\mu \mathrm{g} / \mathrm{l})$ & & & \\
$\quad 100-500$ & 1.000 & & \\
$\quad \leq 100$ & 2.408 & $0.437,13.258$ & 0.313 \\
$\quad \geq 500$ & 0.822 & $0.267,2.532$ & 0.732 \\
\hline
\end{tabular}

MWI, median water iodine; $\mathrm{Tg}$, thyroglobulin; $\mathrm{TgAb}$, thyroglobulin antibody; TPOAb, thyroid peroxidase antibody.

* Abnormal TSH values including cases of hypothyroidism, subclinical hypothyroidism, hyperthyroidism and subclinical hyperthyroidism.

† Binary logistic regression analysis was used to calculate the OR and $95 \% \mathrm{Cl}$ for abnormal TSH in lactating women from the three areas.

increase co-operation and supervision to put effective targeted measures of I supplementation in place. Analogously, it is important to prevent excessive I intake through stopping the provision of iodised salt and implementing effective measurements for water improvement (such as finding other drinking water sources with no excessive I) in high water I areas. Public awareness of the adverse effects of inadequate I intake on infant development needs to be enhanced. Further implementation of scientific I supplementation strategies composed of measures that are contextually appropriate, diverse for the general population and specific for the key ones is necessary.

\section{Conclusion}

Excessive I intake may induce subclinical hypothyroidism in lactating women. Moreover, adequate I nutrition is essential for lactating women and infants, especially those living in I-deficient areas. Enhanced monitoring of I status is important to avoid adverse effects of I deficiency or excess, particularly in susceptible populations such as pregnant or lactating women and infants.

\section{Acknowledgements}

The authors are grateful to the organisations that participated in this survey, including the Institute for Prevention and Treatment of Endemic Disease of Shanxi Province and the Center for Disease Control and Prevention of GuangXi Province for technical assistance. They also thank the colleagues from the above-mentioned organisations who took part in the survey for their dedication and support.

This work was supported by the National Natural Science Foundation of China (grant no. 81273012) and Specialized Research Fund for the Doctoral Program of Senior Education (SRFDP, 20122307110010).

The contribution of each author is as follows: H. S. and L. L. designed the study; H. S., L. L., D. W., P. L. F., M. D. W., Q. J., J. L., X. Z. and P. J. conducted the research; L. L. analysed the data; L. L., D. W. and P. L. wrote the paper; H. S. took the primary responsibility for final content of the manuscript. All the authors read and approved the final version of the manuscript.

There are no conflicts of interest to declare.

The authors have nothing to disclose.

\section{References}

1. Hetzel BS, Potter BJ \& Dulberg EM (1990) The iodine deficiency disorders: nature, pathogenesis and epidemiology. World Rev Nutr Diet 62, 59-119.

2. WHO/UNICEF/ICCIDD (2007) Assessment of Iodine Deficiency Disorders and Monitoring Their Elimination, A Guide For Program Managers, 3rd ed. Geneva: WHO, WHO/ $\mathrm{NDH} / 01.1$.

3. Melse-Boonstra A \& Jaiswal N (2010) Iodine deficiency in pregnancy, infancy and childhood and its consequences for brain development. Best Pract Res ClinEndocrin Metab 24, 29-38.

4. Dorea JG (2002) Iodine nutrition and breast feeding. J Trace Elem Med Biol 16, 207-220.

5. Liu P, Liu L, Shen H, et al. (2014) The standard, intervention measures and health risk for high water iodine areas. PLOS ONE 9, e89608.

6. Chung HR, Shin $\mathrm{CH}$, Yang SW, et al. (2009) Subclinical hypothyroidism in Korean preterm infants associated with high levels of iodine in breast milk. J Clin Endocrinol Metab 94, 4444-4447.

7. Shen H, Liu S, Sun D, et al. (2011) Geographical distribution of drinking-water with high iodine level and association between high iodine level in drinking-water and goitre: a Chinese national investigation. Br J Nutr 106, 243-247.

8. Sun D, Xiao D \& Liu S (2011) The Monitoring of Iodine Deficiency Disorders of China in 2011. Beijing: People's Medical Publishing House.

9. Shen H, Sui X, Ge X, et al. (2009) Delimitation for the Endemic Areas of Iodine Deficiency Disorders (IDD). Beijing: National Standard of China, GB 16005-2009.

10. Liu D, Zhao J, Zhu H, et al. (2003) Determination and Classification of the Areas of High Water Iodine and the Endemic Areas of Iodine Excess Goiter. Beijing: National Standard of China, GB/T1 9380-2003.

11. Yan Y, Zhang Y, Liu L, et al. (2006) Method for Determination of Iodine in Urine By $\mathrm{As}^{3+}-\mathrm{Ce}^{4+}$ Catalytic Spectrophotometry. Beijing: Health Standard of China, WS/T 107-2006.

12. Tong Y \& Huo J (2012) General Test Method in Salt Industry-Determination of Iodine. Beijing: National Standard of China. GB/T 13025.7-2012.

13. Liu L, Li S, Li X, et al. (2008) Method for Determination of Iodine in Foodstuff by As(III)-Ce ${ }^{4+}$ Catalytic Spectrophotometry. Beijing: Health Standard of China. WS 302-2008.

14. Liu S, Chen Z, Jia Q, et al. (2007) Diagnostic Criterion of Endemic Goiter. Beijing: Health Standard of China, Ws 276-2007.

15. Laurberg P, Andersen S, Knudsen N, et al. (2002) Thiocyanate in food and iodine in milk: from domestic animal feeding to improved understanding of cretinism. Thyroid $\mathbf{1 2}$, 897-902.

16. Yan YQ, Chen ZP, Yang XM, et al. (2005) Attention to the hiding iodine deficiency in pregnant and lactating women after universal salt iodization: a multi-community study in China. J Endocrinol Invest 28, 547-553.

17. Yu J, Liu P, Shen $\mathrm{H}$, et al. (2011) The inhabitant's iodine nutrition status of some coastal areas in China: a crosssectional study. Chin J Endemiolog 30, 594-597. 
18. Laurberg P, Pedersen KM, Hreidarsson A, et al. (1998) Iodine intake and the pattern of thyroid disorders: a comparative epidemiological study of thyroid abnormalities in the elderly in Iceland and in Jutland, Denmark. J Clin Endocrinol Metab 83, 765-769.

19. The State Council of the People's Republic of China (1994) Control Iodised Salt Management Regulations for IDD. Beijing: SCPRC (in Chinese).

20. Neville MC, Keller R, Seacat J, et al. (1988) Studies in human lactation: milk volumes in lactating women during the onset of lactation and full lactation. Am J Clin Nutr 48, 1375-1386.

21. Delange F (2007) Iodine requirements during pregnancy, lactation and the neonatal period and indicators of optimal iodine nutrition. Public Health Nutr 10, 1571-1580.

22. Semba RD \& Delange F (2001) Iodine in human milk: perspectives for infant health. Nutr Rev 59, 269-278.

23. Wang Y, Zhang Z, Ge P, et al. (2009) Iodine status and thyroid function of pregnant, lactating women and infants $(0-1 \mathrm{yr})$ residing in areas with an effective Universal Salt Iodization program. Asia Pac J Clin Nutr 18, 34-40.

24. Caldwell KL, Jones R \& Hollowell JG (2005) Urinary iodine concentration: United States National Health and Nutrition Examination Survey 2001-2002. Thyroid 15, 692-699.
25. Wang W, Jin Y, Teng W, et al. (2002) Research of epidemiology of serum TSH levels of healthy people in area with different iodine intake in China. Chin J Endocrinol Metab 18, 355-356.

26. Azizi F \& Smyth P (2009) Breastfeeding and maternal and infant iodine nutrition. Clin Endocrinol (Oxf) 70, 803-809.

27. Meng F, Zhao R, Liu P, et al. (2013) Assessment of iodine status in children, adults, pregnant women and lactating women in iodine-replete areas of China. PLOS ONE 8, e81294.

28. Ma ZF \& Skeaff SA (2014) Thyroglobulin as a biomarker of iodine deficiency: a review. Thyroid 24, 1195-1209.

29. Zimmermann MB, Aeberli I, Andersson M, et al. (2013) Thyroglobulin is a sensitive measure of both deficient and excess iodine intakes in children and indicates no adverse effects on thyroid function in the UIC range of $100-299 \mu \mathrm{g} / / \mathrm{L}$ : a UNICEF/ICCIDD study group report. J Clin Endocrinol Metab 98, 1271-1280.

30. Teng W, Shan Z, Teng X, et al. (2006) Effect of iodine intake on thyroid diseases in China. N Engl J Med 354, 2783-2793.

31. Sang ZN, Wei W, Zhao N, et al. (2012) Thyroid dysfunction during late gestation is associated with excessive iodine intake in pregnant women. J Clin Endocrinol Metab 97, E1363-E1369. 